\title{
Association of Antiplatelet and Anticoagulant Treatment in Patients with Mechanical Prosthetic Heart Valves. Data from the Observational Multicentre PLECTRUM Study
}

\author{
Daniela Poli ${ }^{*}$, Emilia Antonucci ${ }^{2}$, Vittorio Pengo ${ }^{3}$, Eugenio Bucherini ${ }^{4}$, Corrado Lodigiani $^{5}$, \\ Doris Barcellona ${ }^{6}$, Gualtiero Palareti $^{2}$, Sophie Testa $^{7}$ \\ ${ }^{1}$ Center of Atherothrombotic Disease, Azienda Ospedaliero-Universitaria Careggi, Firenze, Italy \\ ${ }^{2}$ Fondazione Arianna Anticoagulazione, Bologna, Italy \\ ${ }^{3}$ Department of Cardiac Thoracic and Vascular Sciences, Thrombosis Centre, University of Padova, Italy \\ ${ }^{4}$ SSA Angiologia e Medicina Vascolare -AUSL Romagna, Ravenna, Italy \\ ${ }^{5}$ Thrombosis and Hemorrhagic Diseases Unit, Cardiovascular Department Humanitas Clinical and Research Center \\ and Humanitas University, Rozzano-Milano, Italy \\ ${ }^{6}$ Centro Emostasi, Azienda Ospedaliero Universitaria, Cagliari, Italy \\ ${ }^{7}$ Haemostasis and Thrombosis Centre, ASST Cremona, Italy
}

"Corresponding Author: Daniela Poli, MD, Centro Trombosi, Viale Morgagni, 85-50134, Firenze, Italy, Tel.: +0039-055-7945453; E-mail: polida@ aou-careggi.toscana.it

Received: 30 December 2020; Accepted: 12 January 2021; Published: 02 February 2021

Citation: Daniela Poli, Emilia Antonucci, Vittorio Pengo, Eugenio Bucherini, Corrado Lodigiani, Doris Barcellona, Gualtiero Palareti, Sophie Testa. Association of antiplatelet and anticoagulant treatment in patients with Mechanical Prosthetic Heart Valves. Data from the observational multicentre PLECTRUM Study. Cardiology and Cardiovascular Medicine 5 (2021): 123-133.

\section{Abstract}

Background: Patients with a mechanical prosthetic heart valve (MHV) need vitamin $\mathrm{K}$ antagonist (VKA) treatment, due to the high thrombotic risk. Guidelines disagree in relation to the need of lowdose aspirin associated treatment (VKA+apl). In
PLECTRUM study, we evaluate the number and characteristics of patients treated with VKA+apl, their bleeding and thrombotic risk.

Methods: Observational retrospective multicenter study among Centers affiliated to the Italian 
Federation of Anticoagulation Clinics (FCSA) on patients with MHV.

Results: 237/2051 (11.5\%) enrolled patients were treated with VKA + apl. These patients were older than patients on single VKA treatment, more frequently males, have a higher rate of cardiovascular risk factors, higher prevalence of coronary artery disease, peripheral arterial obstructive disease, Stroke/transient ischemic attack (TIA), heart failure, and atrial fibrillation. Instead, no difference was found between patients on VKA and patients on VKA + apl in relation to the site of valvular implantation (aortic, mitralic or mitro-aortic) and to the intensity of anticoagulation. The rate of major bleedings, of Stroke/TIA and of death were higher in patients on VKA +apl with respect to patients treated with VKA.

Conclusions: Patients with MHV followed in Italian Anticoagulation Clinics usually receive single VKA treatment. VKA +apl is limited to patients with concomitant arterial disease or at high cardiovascular risk. VKA +apl did not reduce the stroke risk of these patients and is associated with a trend to an increased bleeding risk.

Keywords: Mechanical heart valves; Anticoagulation; Vitamin $\mathrm{K}$ antagonists; Aspirin; Stroke; Bleeding

\section{Introduction}

The burden of valvular heart disease, due to the high incidence of rheumatic heart disease in developing countries and of degenerative valve disease in western countries, is a growing clinical problem [1]. The implantation of a prosthetic valve, mechanical or biological, is frequently required in these patients.
The two types of valves differ mainly in duration and thrombogenicity, being mechanical heart valves more durable but more thrombogenic than bio-prostheses. Due to the higher risk of thrombosis and systemic embolism, patients with mechanical heart valves (MHV) require long-term oral anticoagulant therapy with a vitamin $\mathrm{K}$ antagonists (VKA) [2]. The StarrEdwards caged-ball valve was the first prosthetic heart valve implanted in 1960 [3]. This valve has been subsequently replaced first by tilting disc valves, and eventually by bi-leaflet valves. Nowadays bi-leaflet valves are by far the most commonly used, due to their lowest thrombogenic profile [1]. The thrombotic risk associated with the prosthetic valves depends also on the site of valve implantation, with mitral valves more thrombogenic than aortic valves [4]. The need for long-life VKA anticoagulation is unanimously recommended, with a higher intensity of anticoagulation (International Normalized Ratio INR- target of 3.0) in patients with mitral valve replacement or aortic valve replacement with additional risk factors for thromboembolism, and less intense anticoagulation (INR target 2.5) in patients with low-risk aortic valve [4, 5]. However, international guidelines give different recommendations with regard to the use of associated aspirin treatment. The European Society of Cardiology (ESC) recommends to limit this association to patients with other indications to antiplatelet drugs, such as coronary artery disease, or when a thromboembolic event occurs despite well conducted anticoagulation [4]. Instead, the American College of Cardiology/American Heart Association (ACC/AHA) recommends the association of low-dose aspirin in all patients with MHV [6]. However, this recommendation is mainly based on few studies, conducted more than thirty years ago, therefore 
including patients with the older type of highly thrombogenic valve [7-8]. The population of patients with MHV currently found in clinical practice is essentially limited to patients carrying be-leaflet valves, with lower thrombogenicity profile [1,9]. Moreover, the increased bleeding risk of the association of aspirin to VKA is the principal concern. Therefore, the optimal therapeutic strategy for patients with MHV is still an open issue.

The purpose of this study is to describe the Italian clinical practice on this matter, analyzing data of the previously published observational retrospective multicenter PLECTRUM study [9]. In this sub-study we are aimed to evaluate if differences exist between patient treated with VKA only and patients treated with VKA and antiplatelet drugs (VKA+apl); we recorded the bleeding and thrombotic risk of patients according to the type of treatment.

\section{Methods}

The FCSA-START Valve Study (PLECTRUM) is an observational retrospective multicenter study performed within the Italian $\underline{\text { Survey on anTicogulated }}$ patients $\quad \underline{R}$ egisTer (START Register) (NCT02219984) [10], and conducted among 33 Centers affiliated to the Italian Federation of Anticoagulation Clinics (FCSA). Centers were asked to select from their databases patients in whom a mechanical heart valve prosthesis or a bio-prosthesis was implanted after 1990 and who were followed for the management of anticoagulation. 3029 patients were enrolled in the study, of whom 2357 patients received MHV implantation. Methods have been previously described [9]. Briefly, participating Centres followed patients for the management of anticoagulation after hospital discharge. All Centres adhere to FCSA and participate in the specifically designed laboratory external quality control program, which is run 3 times per year and uses lyophilized plasma samples obtained from anticoagulated patients [11]. At enrollment patients' demographic information and clinical data were collected. The Centre provides regular INR measurements, prescribes the daily VKA dosages and schedules the date for the subsequent visits.

According to the routine practice of the participating centers, follow-up visits were scheduled every 2 to 4 weeks for INR monitoring. Patients who missed check-ups for $>2$ months were contacted (personally or through their family or general practitioner), and the reason for interrupting treatment monitoring was recorded. The occurrence of bleeding or thrombotic complications during follow-up is recorded. Major bleeding was defined accordingly with the definition in use in Italian Centres in the study period [12]. The follow-up was stopped at patient's death, after the occurrence of the first adverse event or when a patient was no longer monitored by the participating Center.

The quality of anticoagulation control, was calculated as Time in Therapeutic Range (TTR) by using the linear interpolation method by Rosendaal et al. [13]. TTR was analyzed considering the INRs recorded in the last year of follow-up, and the intended anticoagulation ranges referred to the last year of follow-up for the examined patients. The work was conducted according to declaration of Helsinki. All local Institutional Committees on Human Experimentation have approved the research protocol. 


\subsection{Statistical analysis}

Baseline characteristics were summarized with descriptive statistics. Categorical variables were reported as counts and percentages and continuous variables were expressed as median and interquartile range (IQR). Incidence rates of adverse events were calculated as the number of events per 100 patientyears of observation, and rate ratios were given with their $95 \%$ confidence intervals (CI).

For this calculation, observation started at the beginning of the follow-up and ended when patients experienced either death, a major outcome or stop regular monitoring by the participating centre. Analyses were performed with the Fisher exact test (for categorical data), the unpaired t test (for normally distributed data), and the Mann-Whitney test (for nonnormally distributed data).

To evaluate the association of conditions to guide the prescription of antiplatelet drugs in association with VKA a lostic regression analysis was performed. All variables found to be significant at univariable analysis were subsequently entered into a multivariable analysis. Risk was expressed as odds ratio (OR) with 95\% CI. A 2-sided value of $\mathrm{p}<0.05$ was chosen for statistical significance.

We used the SPSS version 25 software (SPSS Inc, Chicago, IL, USA) and the Stata version 14 software (Stata Corp, College Station, TX) for Windows for data processing.

\section{Results}

In the PLECTRUM Study 2357 patients with MHV were included. Clinical characteristics of this cohort are resumed in Table 1. Information on associated treatment with antiplatelet drugs was available for 2051/2357 (87.0\%) patients. Among these patients, $237 / 2051(11.5 \%)$ patients were on treatment with VKA + apl: $209(88.2 \%)$ patients were treated with aspirin 75-100 mg, $17(7.2 \%)$ patients with clopidogrel $75 \mathrm{mg}$, and $11(4.6 \%)$ patients were on dual antiplatelet treatment with aspirin $100 \mathrm{mg}$ and clopidogrel $75 \mathrm{mg}$.

The characteristics of patients treated only with VKAs and of patients treated with VKA+ apl are reported in Table 2. Patients on treatment with VKA+ apl are older than patients treated only with VKA, more frequently were males, and have a significantly higher rate of risk factors for cardiovascular disease, such as hypertension and diabetes mellitus. Similarly, they showed higher prevalence of coronary artery disease, peripheral arterial obstructive disease, history of previous Stroke/transient ischemic attack (TIA), heart failure and atrial fibrillation.

Instead, no difference was found between patients on VKA only and patients on VKA + apl in relation to the site of valvular implantation (aortic, mitralic or mitro-aortic) and to the intended INR therapeutic range. 


\begin{tabular}{|c|c|}
\hline All & 2357 \\
\hline Males & $1301(55.2)$ \\
\hline Median age at implantation (IQR) & $59.0(49.7-65.7)$ \\
\hline \multicolumn{2}{|l|}{ Site of prosthesis valve, $\mathrm{n}(\%)$} \\
\hline Aortic & $1408(59.7)$ \\
\hline Mitral & $682(28.8)$ \\
\hline Mitral-Aortic & $267(11.5)$ \\
\hline \multicolumn{2}{|l|}{ Vitamin $\mathrm{K}$ antagonist $\mathrm{n}(\%)$} \\
\hline Warfarin & $1929(81.8)$ \\
\hline Acenocoumarol & $428(18.2)$ \\
\hline \multicolumn{2}{|l|}{ Follow-up } \\
\hline Median follow-up (years) (IQR) & $9.7(5.0-14.1)$ \\
\hline Total follow-up (pt-yrs) & 24,081 \\
\hline \multicolumn{2}{|l|}{ Antithrombotic Treatment $(2051 / 2357), \mathrm{n}(\%)$} \\
\hline VKA & $1814(88.5)$ \\
\hline VKA+ antiplatelet treatment & $237(11.5)$ \\
\hline
\end{tabular}

Table 1: Characteristics of the whole cohort. 


\begin{tabular}{|c|c|c|c|}
\hline & VKA & VKA + antiplatelet & p value \\
\hline Number (\%) & 1814 (88.5) & $237(11.5)$ & - \\
\hline Males & $990(54.5)$ & $150(63.6)$ & 0.008 \\
\hline Median Age at implantation (IQR) & $58.4(48.7-65.3)$ & $60.0(54.9-67.4)$ & 0.000 \\
\hline \multicolumn{4}{|l|}{ Follow-up } \\
\hline Median follow-up (years) (IQR) & $9.6(5.1-13.9)$ & $9.0(4.0-13.6)$ & 0.4 \\
\hline Total follow-up (pt-yrs) & 18423 & 2148 & - \\
\hline Dual antiplatelet therapy & - & $11(4.6)$ & - \\
\hline \multicolumn{4}{|l|}{ Co-morbidities } \\
\hline Hypertension & $1130(62.3)$ & $188(79.3)$ & 0.000 \\
\hline Diabetes Mellitus & $225(12.4)$ & $56(23.6)$ & 0.000 \\
\hline Atrial Fibrillation & $669(36.9)$ & $109(46.0)$ & 0.008 \\
\hline Heart Failure & $120(6.6)$ & $34(14.3)$ & 0.004 \\
\hline Previous Stroke/TIA & $122(6.7)$ & $36(15.2)$ & 0.000 \\
\hline Coronary Artery disease & $165(9.1)$ & $105(44.3)$ & 0.000 \\
\hline Peripheral artery disease & $56(3.1)$ & $50(21.1)$ & 0.000 \\
\hline Carotid artery disease & $59(3.3)$ & $50(21.1)$ & 0.000 \\
\hline Renal failure & $25(1.4)$ & - & na \\
\hline No CAD or POAD & $1374(75.7)$ & $71(30.0)$ & 0.000 \\
\hline Site of valve implantation & - & - & 0.2 \\
\hline Aortic & $1093(60.3)$ & $152(64.1)$ & - \\
\hline Mitralic & $517(28.5)$ & $63(26.6)$ & - \\
\hline Mitro-Aortic & $204(11.2)$ & $22(9.3)$ & - \\
\hline \multicolumn{4}{|l|}{ Intended INR therapeutic range } \\
\hline $2.0-3.0$ & $485(26.7)$ & $70(29.5)$ & 0.2 \\
\hline 2.5-3.5 or higher & $1329(73.3)$ & $167(70.5)$ & 0.2 \\
\hline Time spent in therapeutic range $(\%)$ & $60(47-74)$ & $58(44-70)$ & 0.12 \\
\hline
\end{tabular}

Table 2: Characteristics of patients on VKA and VKA + antiplatelet treatment. 
We performed univariable and multivariable analysis to evaluate the conditions associated with the prescription of antiplatelet drugs in association with VKA (Table 3). The presence of hypertension, and the history of coronary artery disease, Stroke/TIA, peripheral arterial obstructive disease, and carotid artery disease, were independently associated with VKA+apl treatment.

\begin{tabular}{|l|l|l|l|l|}
\hline & OR (95\% CI) & P value & OR (95\% CI) & P value \\
\hline Male sex & $1.5(1.1-1.9)$ & 0.008 & $1.25(0.9-1.8)$ & 0.2 \\
\hline Hypertension & $2.4(1.7-3.3)$ & 0.000 & $1.8(1.1-3.0)$ & 0.02 \\
\hline Diabetes Mellitus & $2.2(1.6-3.1)$ & 0.000 & $1.23(08-1.9)$ & 0.3 \\
\hline Atrial Fibrillation & $1.5(1.1-1.9)$ & 0.007 & $1.4(1.0-2.1)$ & 0.06 \\
\hline Heart Failure & $1.9(1.2-2.9)$ & 0.003 & $1.2(0.7-1.9)$ & 0.6 \\
\hline Previous Stroke/TIA & $2.5(1.7-3.7)$ & 0.000 & $1.8(1.0-3.2)$ & 0.04 \\
\hline Coronary Artery disease & $8.1(6.0-10.9)$ & 0.000 & $6.5(4.4-9.6)$ & 0.000 \\
\hline Peripheral Artery disease & $8.3(5.5-12.5)$ & 0.000 & $2.9(1.6-5.5)$ & 0.001 \\
\hline Carotid Artery disease & $8.1(5.4-12.2)$ & 0.000 & $4.3(2.6-7.3)$ & 0.000 \\
\hline Absence of Artery disease & $0.14(0.1-0.2)$ & 0.000 & $0.7(0.3-1.3)$ & 0.3 \\
\hline
\end{tabular}

Table 3: Univariate and multivariate analysis.

During the follow-up, death occurred in 164 patients (rate $0.80 / 100$ pt-yrs); thromboembolic events occurred in 150 patients (rate $0.73 \times 100$ pt-yrs), and major bleedings in 230 patients (rate 1.12x100 pt-yrs). The rate of major bleedings was higher among patients on VKA +apl with respect to patients on VKA, even if this difference was not statistically significant (OR 1.3; 95\% CI 0.8-1.8). Similarly, the rate of Stroke/TIA and of death were higher among patients on VKA +apl with respect to patients on VKA (OR 2.9; 95\% CI 2.2-4.2 and OR 1.7; 95\% CI 1.1-2.6, respectively) (Table 4). Dual antiplatelet therapy with both aspirin and clopidogrel was recorded in 11/237 (4.6\%) patients. Among these patients major bleeding occurred in 4/11 (36.4\%) (rate $3.2 \times 100 \mathrm{pt}-\mathrm{yrs})$, and death was recorded in $3 / 11$ (27.3\%) (rate 2.4 x 100 pt-yrs).

\begin{tabular}{|l|l|l|l|l|}
\hline & VKA (rate) & VKA + apl (rate) & RR (95\% CI) & p value \\
\hline Major bleeding & $199(1.08)$ & $31(1.44)$ & $1.25(0.8-1.8)$ & 0.2 \\
\hline ICH & $44(0.24)$ & $6(0.28)$ & $1.2(0.4-2.7)$ & 0.7 \\
\hline GI & $58(0.32)$ & $13(0.47)$ & $1.9(1.0-3.5)$ & 0.04 \\
\hline Stroke & $110(0.60)$ & $40(1.86)$ & $2.9(2.0-4.2)$ & 0.000 \\
\hline Death & $137(0.74)$ & $27(1.25)$ & $1.7(1.1-2.6)$ & 0.02 \\
\hline
\end{tabular}

Table 4: Adverse events in patients on VKA or VKA + apl treatment 


\section{Discussion}

The principal finding of this observational study is the low number of associated antiplatelet treatment among Italian patients on long-term anticoagulation with VKA after MHV implantation. The study was conducted on a large cohort of MHV patients, with a long follow-up period, and confirms that Italian Centres treat these patients mainly with single VKA treatment, in keeping with Italian (FCSA) and European Guidelines [4]. These guidelines recommend to limit the association of low-dose aspirin to VKA to patients with other indications to antiplatelet drugs, such as coronary artery disease, or in case of thrombotic complications inspite of a well conducted anticoagulation. On the contrary, American Guidelines recommend to add aspirin to all patients with MHV implantation [6]. However, this recommendation is essentially based on two studies, conducted more than 30 years ago, including small groups of patients $[14,15]$. In particular in the study of Meschengieser et al. about 25\% of patients received a Starr-Edwards caged-ball valve, a prosthetic type of valve with a high thrombotic risk profile, that is now not more implanted [15]. Moreover, in the study of Turpie et al. [14] a large number of patients with coronary artery disease were included, and the benefit found in the group of patients treated with aspirin was mainly due to the reduction of cardiovascular death. In this latter study, the risk of major bleedings was similar between patients with and without aspirin treatment. However, when we consider the incidence of intracerebral hemorrhage, this severe complication occurred in 8 patients on combined VKA and aspirin treatment, and in 3 patients treated only with VKA. Instead, the study of Meschengieser et al. reported a higher bleeding risk in the group of patients maintained at low-intensity anticoagulation with aspirin, with respect to patients treated with warfarin at highintensity level [15].

Differently from these studies, we enrolled patients who underwent implantation after 1990, when the use of the oldest, highly thrombogenic Starr-Edward valves had already been largely abandoned in favor of less thrombogenic valves [9].

Later, Laffort $\mathrm{P}$ et al. published the results of a randomized trial comparing single VKA treatment with VKA +apl in a small group of patients who received between 1994 and 1996 a be-leaflet St. Jude prosthesis with low thrombogenic profile [16]. The study found a reduction of thromboembolic events in the first year after MHV implantation in the group of patients receiving VKA+apl. However, no reduction in mortality was found and an increase of bleeding complications was recorded. More recently, a prospective randomized study was performed in China to compare VKA treatment vs VKA + apl in a small group of MHV patients [17]. In this study, a lower thrombotic risk was found in patients on VKA+apl with respect to VKA treatment, without higher bleeding risk. However, in this study patients were maintained at very low-intensity anticoagulation level (INR 1.8-2.5), and a very low quality of anticoagulation was obtained, with less than $40 \%$ of patients achieving good INR control. Differently from this Chinese experience, the quality of anticoagulation obtained in MHV patients treated in European countries is higher [7, 9, 18]. An Italian pilot randomized study published in 2008 reported that low-intensity VKA treatment (INR 2.0-3.0) plus aspirin was as effective and safe as high intensity VKA treatment (INR target 3.7) [18]. However, the 
study was limited to a small group of patients, and to the first six months after prosthesis implantation, similarly to the study of Laffort et al [16]. Moreover, the high intensity VKA treatment used in the study is now not recommended, due the difficulties in maintain adequate anticoagulation, and to the associated bleeding risk $[4,6]$.

In our study, the addition of aspirin to VKA is associated with a trend to an increase of major bleeding risk, without a difference in the occurrence of intracerebral bleedings, whereas gastro-intestinal bleedings were increased. These results are in keeping also with data obtained in several studies when aspirin is added to VKA in other clinical settings, such as atrial fibrillation [19, 20].

It should be noted that we found that the use of aspirin was also associated with a significant increase in the risk of ischemic stroke. This finding is not surprising when we take in account the higher prevalence of coronary artery disease and of risk factor for ischemic stroke recorded in this group of patients, conditions that favor the clinical decision to add aspirin to warfarin treatment. Interestingly, no difference in the use of aspirin was found in relation to the site of valve implantation, aortic or mitralic, notwithstanding the different thrombotic risk associated with valve position [4].

\section{Study Limitation}

We are aware of limitations of our study. Firstly, this is an observational retrospective cohort study, and therefore we could not adjust for clinical information that were not recorded. In particular, the duration of the concomitant use of antiplatelet drugs associated to VKAs is not reported in the data set, therefore we cannot exclude that the time of exposure to combined VKA+apl treatment could be different from total observational period.

Strengths of our study are however the large number of patients included, the multicenter design, and the prospective recording of anticoagulation history and of the adverse events in electronic data bases routinely done by participating Centres.

\section{Conclusion}

Patients with MHV followed in Italian Centres for the management of anticoagulation, usually receive single VKA treatment. The association with low-dose aspirin is limited to patients with concomitant arterial disease or at high cardiovascular risk. The associated treatment did not reduce the stroke risk of these patients and is associated with a trend to an increased bleeding risk.

\section{Aknowledgments}

The work was supported by the Arianna Anticoagulation Foundation that supported the START Register. The Foundation had no role in study design, collection, analysis and interpretation of data, in writing the report, and in the decision to submit the paper for publication.

\section{Conflicts of Interest}

None declared.

\section{References}

1. Sun JC, Davidson MJ, Lamy A, Eikelboom JW. Antithrombotic management of patients with prosthetic heart valves: current evidence and future trends. Lancet 374 (2009): 565576. 
2. Whitlock RP, Sun JC, Fremes SE, Rubens FD, Teoh KH. Antithrombotic and Thrombolytic Therapy for Valvular Disease: Antithrombotic Therapy and Prevention of Thrombosis, 9th ed: American College of Chest Physicians Evidence-Based Clinical Practice Guidelines. Chest 141 (2012): e576S-600S.

3. Starr A, Edwards ML. Mitral replacement: clinical experience with a ball-valve prosthesis. Ann Surg 154 (1961): 726-740.

4. Joint Task Force on the Management of Valvular Heart Disease of the European Society of C, European Association for Cardio-Thoracic. S Vahanian A, Alfieri O, Andreotti F, Antunes MJ, Baron-Esquivias $\mathrm{G}$, et al. Guidelines on the management of valvular heart disease (version 2012). Eur Heart J 33 (2012): 2451-2496.

5. Nishimura RA, Otto CM, Bonow RO, Carabello BA, Erwin JP, et al. 2017 AHA/ACC Focused Update of the 2014 AHA/ACC Guideline for the Management of Patients With Valvular Heart Disease: A Report of the American College of Cardiology/American Heart Association Task Force on Clinical Practice Guidelines. J Am Coll Cardiol (2017).

6. Nishimura RA, Otto CM, Bonow RO, Carabello BA, Erwin JP, et al. 2017 AHA/ACC Focused Update of the 2014 AHA/ACC Guideline for the Management of Patients With Valvular Heart Disease: A Report of the American College of Cardiology/American Heart Association Task Force on Clinical Practice Guidelines. Circulation 135 (2017): e1159-e1195.
7. Labaf A, Svensson PJ, Renlund H, Jeppsson A, Sjalander A. Incidence and risk factors for thromboembolism and major bleeding in patients with mechanical valve prosthesis: A nationwide population-based study. Am Heart J 181 (2016): 1-9.

8. Torella M, Torella D, Chiodini P, Franciulli M, Romano G, et al. LOWERing the INtensity of oral anticoaGulant Therapy in patients with bileaflet mechanical aortic valve replacement: results from the "LOWERING-IT" Trial. Am Heart J 160 (2010): 171-178.

9. Poli D, Antonucci E, Pengo V, Migliaccio L, Testa S, et al. Italian Federation of Anticoagulation C. Mechanical prosthetic heart valves: Quality of anticoagulation and thromboembolic risk. The observational multicenter PLECTRUM study. Int J Cardiol 267 (2018): 68-73.

10. Antonucci E, Poli D, Tosetto A, Pengo V, Tripodi A, et al. The Italian START-Register on Anticoagulation with Focus on Atrial Fibrillation. PLoS One 10 (2015): e0124719.

11. Tripodi A, Chantarangkul V, Akkawat B, Clerici M, Mannucci PM. A partial factor v deficiency in anticoagulated lyophilized plasmas has been identified as a cause of the international normalized ratio discrepancy in the external quality assessment scheme. Thromb Res 78 (1995): 283-292.

12. Palareti G, Leali N, Coccheri S, Poggi M, Manotti C, et al. Bleeding complications of oral anticoagulant treatment: an inceptioncohort, prospective collaborative study (ISCOAT). Italian Study on Complications 
of Oral Anticoagulant Therapy. Lancet 348 (1996): 423-428.

13. Rosendaal FR, Cannegieter SC, Vandermeer FJM, Briet E. A method to determine the optimal intensity of oral anticoagulant therapy. Thromb Haemost 69 (1993): 236237.

14. Turpie AG, Gent M, Laupacis A, Latour Y, Gunstensen J, et al. A comparison of aspirin with placebo in patients treated with warfarin after heart-valve replacement. N Engl J Med 329 (1993): 524-529.

15. Meschengieser SS, Fondevila CG, Frontroth J, Santarelli MT, Lazzari MA. Low-intensity oral anticoagulation plus low-dose aspirin versus high-intensity oral anticoagulation alone: a randomized trial in patients with mechanical prosthetic heart valves. J Thorac Cardiovasc Surg 113 (1997): 910-916.

16. Laffort P, Roudaut R, Roques X, Lafitte S, Deville C, et al. Early and long-term (oneyear) effects of the association of aspirin and oral anticoagulant on thrombi and morbidity after replacement of the mitral valve with the St. Jude medical prosthesis: a clinical and transesophageal echocardiographic study. J Am Coll Cardiol 35 (2000): 739-746.

17. Dong MF, Ma ZS, Ma SJ, Chai SD, Tang $\mathrm{PZ}$, et al. Anticoagulation therapy with combined low dose aspirin and warfarin following mechanical heart valve replacement. Thromb Res 128 (2011): e91e94.

18. Pengo V, Palareti G, Cucchini U, Molinatti M, Del Bono R, et al. Participating centers of Italian Federation of Anticoagulation C. Low-intensity oral anticoagulant plus lowdose aspirin during the first six months versus standard-intensity oral anticoagulant therapy after mechanical heart valve replacement: a pilot study of low-intensity warfarin and aspirin in cardiac prostheses (LIWACAP). Clin Appl Thromb Hemost 13 (2007): 241-248.

19. Dentali F, Douketis JD, Lim W, Crowther M. Combined aspirin-oral anticoagulant therapy compared with oral anticoagulant therapy alone among patients at risk for cardiovascular disease: a meta-analysis of randomized trials. Arch Intern Med 167 (2007): 117-124.

20. Hansen ML, Sorensen R, Clausen MT, FogPetersen ML, Raunso $J$, et al. Risk of bleeding with single, dual, or triple therapy with warfarin, aspirin, and clopidogrel in patients with atrial fibrillation. Arch Intern Med 170 (2010): 1433-1441. 